\title{
Diamond nucleation enhancement by direct low-energy ion-beam deposition
}

\author{
W. J. Zhang, X. S. Sun, H. Y. Peng, N. Wang, C. S. Lee, I. Bello, and S. T. Lee* \\ Center Of Super-Diamond \& Advanced Films (COSDAF) and Department of Physics \& Materials Science, City University of Hong \\ Kong, \\ Hong Kong, China \\ (Received 2 February 1999)
}

\begin{abstract}
Direct ion beam deposition was successfully applied for the nucleation of nanodiamond crystallites on mirror-polished $\mathrm{Si}(001)$ substrates. Low-energy $(80-200 \mathrm{eV})$ argon, hydrocarbon, and hydrogen ions from a Kaufman ion source were used. An amorphous carbon film was deposited on the substrate after ion bombardment. The films were characterized by high-resolution transmission electron microscopy, selected area electron diffraction, secondary electron microscopy, and micro-Raman spectroscopy. At ion doses above 1 $\times 10^{18} \mathrm{~cm}^{-2}$, nanocrystalline diamond particles of 50-100 $\AA$ in diameter were formed in a matrix of amorphous carbon. These diamond nanocrystals served as nucleation centers for subsequent diamond growth by conventional hot filament chemical vapor deposition. The nucleation density depended strongly on the ion dosage, and a nucleation density of $3 \times 10^{9} \mathrm{~cm}^{-2}$ could be achieved under optimized conditions. These results were found very helpful for the evaluation of the mechanism of ion-bombardment-induced nucleation of diamond.
\end{abstract}

\section{INTRODUCTION}

Chemical vapor deposition (CVD) of diamond was thoroughly investigated during the last decade, motivated by the unique set of properties of diamond, which makes it an excellent candidate material for a variety of applications. Diamond growth on existing diamond nuclei is properly understood, and was realized on a variety of substrates by different methods. The nucleation stage of diamond is, however, still not understood, though several methods were found by trail and error studies to initiate the formation of diamond nuclei on nondiamond substrates, very frequently on single crystal Si wafers. These include various ex situ (e.g., prescratching of $\mathrm{Si}$ wafers $^{1,2}$ and ion implantation ${ }^{3}$ ) and in situ [biasenhanced-nucleation $\left.(\mathrm{BEN})^{4}\right]$ processes. Among these enhancement methods, BEN was established as a technique capable of deposition of oriented diamond films on nondiamond substrates, when followed by a subsequent microwave CVD (Refs. 5 and 6) or hot filament CVD (Refs. 7 and 8) growth. Many attempts have been made to understand the BEN process, and different models have been proposed. Yugo and co-workers ${ }^{9,10}$ and Gerber et al. ${ }^{11}$ suggested a shallow ion implantation (subplantation) model, in which $s p^{3}$-bonded carbon clusters, formed by ion implantation, serve as nucleation precursors. Some researchers proposed that an increased amount of reactive hydrocarbon and atomic hydrogen in the plasma and the formation of a carbide surface layer should play the decisive role. ${ }^{12-14}$ Beckmann et al. ${ }^{15}$ noted that this change in the plasma chemistry was too small to account for the nucleation enhancement. Jiang, Schiftman, and Klages ${ }^{16}$ found that the overall temporal evolution of nucleation density agreed well with a surface kinetic model proposed by Tomellini, Polini, and Sessa. ${ }^{17}$ They further suggested that ion bombardment plays a decisive role in the BEN process. Ion bombardment, and thus its effect, are invariably present in the BEN process. ${ }^{18,19}$ In spite of previous studies, the mechanism of ion-bombardmentenhanced nucleation still is not understood.
The ion beam technique is the most direct and effective method to study the effect of ion bombardment on nucleation enhancement. The advantage of the ion beam technique (compared to plasma bias-enhanced techniques) is the easy control of the fluence, species, and energy of the ions incident on the growing surface. Such controllability enables a systematic study of the process of diamond nucleation by ion bombardment. Numerous attempts have been undertaken to deposit diamond on various substrates by means of ion implantation and the mass-selected ion beam method. Lee et $a l .{ }^{20}$ reported that carbon ion implantation at medium energy $(5-25 \mathrm{keV})$ into diamond substrates led to the growth of polycrystalline diamond with a very high density of extended defects. In spite of the many studies of carbon ion implantation into foreign substrates, ${ }^{21-24}$ no definitive evidence for diamond formation was obtained. Recently, Guo et $a l^{25}$ reported the formation of diamond microcrystallites on Si by means of hydrocarbon and hydrogen ion bombardment, but only x-ray photoelectron spectroscopic characterization was given to support the results.

In this paper, we used a direct two-step process to study the effect of ion bombardment on diamond nucleation. In the first step, hydrocarbon and hydrogen ion bombardment was used to induce nucleation on mirror-polished (001) Si substrates. In the second step, diamond films were subsequently deposited on the ion-bombarded substrates by conventional hot filament chemical vapor deposition (HFCVD). Fieldemission gun scanning electron microscopy (FEG-SEM), high-resolution transmission electron microscopy (HRTEM), selective area electron diffraction (SAED), and micro-Raman spectroscopy were used to characterize the ion-bombarded substrates and the deposited films.

The present paper gives definitive evidence for diamond nucleation on nontreated $\mathrm{Si}(100)$ substrates using direct ion beam bombardment, with ions generated in a Kaufman ion source fed by a $\mathrm{CH}_{4}: \mathrm{Ar}: \mathrm{H}_{2}$ gas mixture. This nucleation treatment was found efficient to further grow diamond by conventional HFCVD, achieving a high nucleation density. 


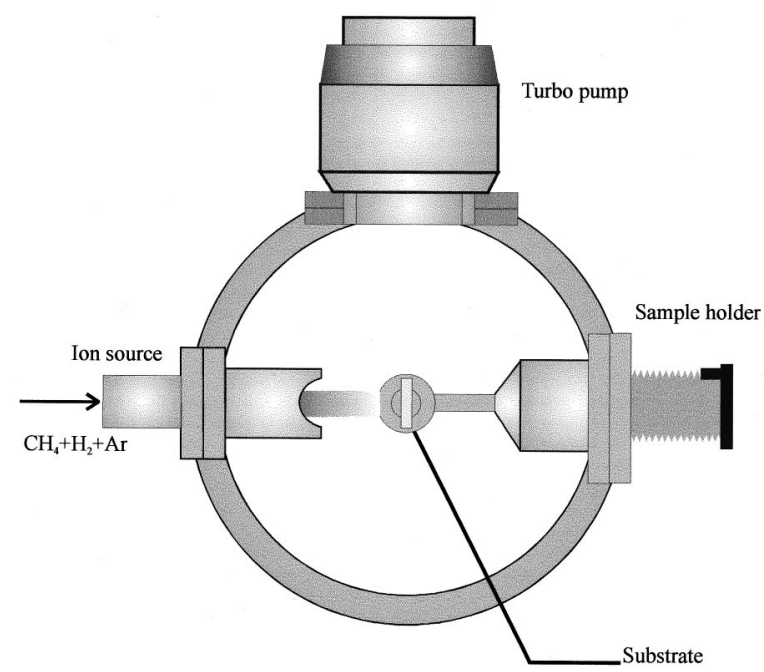

FIG. 1. Schematic diagram of the ion beam deposition system equipped with a Kaufman ion source.

The present systematic study was found very helpful in discussing the ion-beam-induced nucleation mechanism.

\section{EXPERIMENT DETAILS}

The experiments were divided into two steps: (1) nucleation enhancement by ion beam deposition, and (2) subsequent deposition of diamond films by HFCVD. The first step was carried out in a vacuum chamber equipped with a Kaufman ion source (3.0-1500-1000, Ion Tech, Inc. USA), which is shown in Fig. 1. The base pressure of the reactor was $\sim 2 \times 10^{-8}$ Torr. Mirror-polished $n$-type (001) Si wafers were used as substrates. Before ion beam deposition, Si wafers were first rinsed with acetone and ethanol, and then etched with $5 \%$ HF solution (diluted by deionized water) for 1 min. A mixture of methane, hydrogen, and argon $\left(\mathrm{CH}_{4}: \mathrm{H}_{2}: \mathrm{Ar}=1: 14: 4\right)$ was introduced into the ion source as the working gas at $2 \mathrm{sccm}$ and $1 \times 10^{-3}$ Torr. The use of argon is for stabilizing the ion source, which cannot be operated steadily with only $\mathrm{CH}_{4}$. The ions were directed to bombard the substrate perpendicularly. The total ion dosage (measured with a Faraday cup) was varied from $10^{17}$ to $10^{19} \mathrm{~cm}^{-2}$ for ion energy between 80 and $200 \mathrm{eV}$. The substrate temperature was maintained at $780{ }^{\circ} \mathrm{C}$ as measured with an infrared pyrometer. In the second step, the ionbombarded samples were placed in a HFCVD reactor for subsequent growth. The HFCVD growth conditions are as follows: a gas composition of $\mathrm{CH}_{4} / \mathrm{H}_{2}=2: 98$, a reactant gas pressure of 30 Torr, a filament temperature $2100{ }^{\circ} \mathrm{C}$, and a substrate temperature of $850^{\circ} \mathrm{C}$. To investigate the effect of ion bombardment on nucleation, the growth time was kept constant at 15 min HRTEM (Philips FEG CM200 operated at $200 \mathrm{keV}$ ), SAED, FEG-SEM (Philips FEG XL30), and micro-Raman spectroscopy were used to characterize the samples.

\section{RESULTS AND DISCUSSION}

\section{A. Diamond nucleation density vs ion dose and species}

Figure 2 shows a SEM surface morphology of the sample

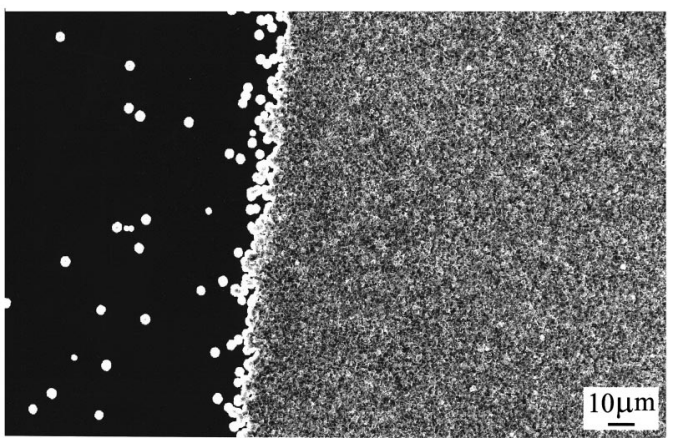

FIG. 2. SEM surface morphology of the sample after conventional deposition by HFCVD for $20 \mathrm{~h}$ on the Si substrate with (right side) and without (left side) prior ion bombardment.

after HFCVD for $20 \mathrm{~h}$ on a partially ion-bombarded Si substrate. Nucleation enhancement by ion bombardment can be clearly seen. On the right-hand side of the figure, where the substrate was prebombarded with $150-\mathrm{eV}$ ions at a dosage of $10^{19} \mathrm{~cm}^{-2}$, it can be seen that a continuous diamond film was deposited with a nucleation density of $>10^{8} / \mathrm{cm}^{2}$. On the left-hand side of the figure, the substrate was covered during the bombardment process, so it received no ion bombardment. On this part of the substrate only a few disperse diamond grains were deposited. The above observation gave direct and definitive confirmation that the bombardment of hydrogen/hydrocarbon/argon ions can indeed induce diamond nucleation enhancement on a mirror-polished Si substrate at a specific ion dose.

To study the effects of ion bombardment upon diamond nucleation, the ion-bombarded substrates were subject to subsequent growth by HFCVD for $15 \mathrm{~min}$ so as to enlarge the grain size for ease of observation. A typical SEM surface morphology of the sample after growth is shown in Fig. 3. The sample was bombarded with $\left(\mathrm{CH}_{4}: \mathrm{Ar}: \mathrm{H}_{2}\right)$ ions to a dose of $1 \times 10^{19} \mathrm{~cm}^{-2}$. Figure 3 shows that the diamond grains had an average size of about $150-200 \mathrm{~nm}$. The grains have not coalesced to form a continuous film, so the nucleation density can readily be counted to be about $3 \times 10^{9} \mathrm{~cm}^{-2}$. The same procedure was performed for samples bombarded with ions of $80,100,150$, and $200 \mathrm{eV}$ at doses ranging from $10^{17}$ to $10^{19} \mathrm{~cm}^{-2}$. The corresponding nucleation density versus the ion dosage at $150 \mathrm{eV}$ is shown in Fig. 4, where it can be seen that $10^{18} \mathrm{~cm}^{-2}$ appears to be the threshold dose for ion-

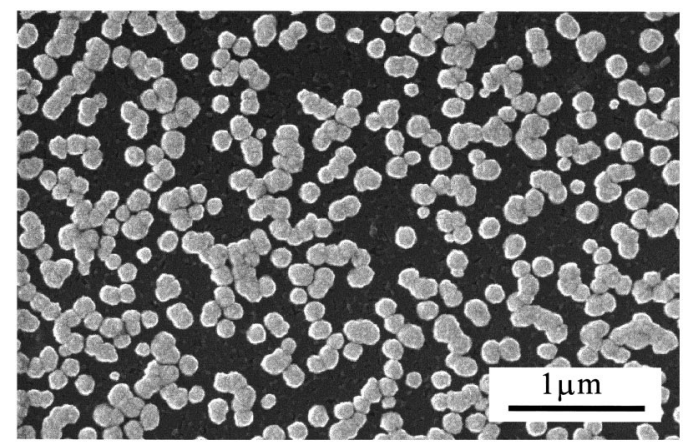

FIG. 3. SEM image of a sample after bombarded with $10^{19}$ ions $\mathrm{cm}^{-2}$ of $\left(\mathrm{CH}_{x}+\mathrm{H}_{x}+\mathrm{Ar}\right)$ and subsequent growth by HFCVD for $15 \mathrm{~min}$. 


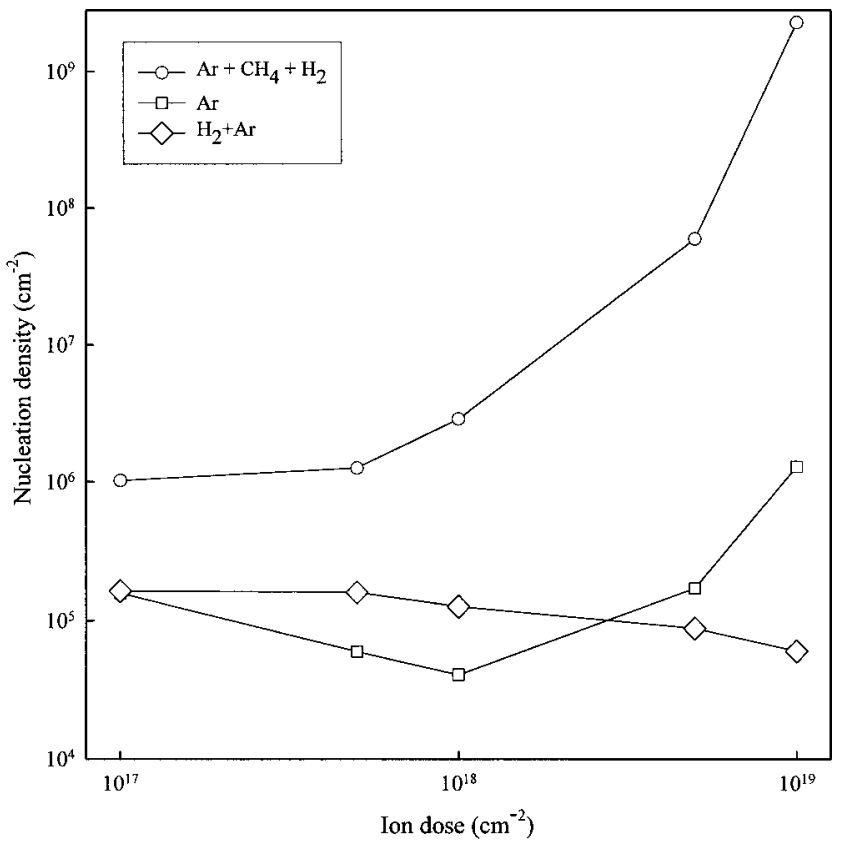

FIG. 4. Diamond nucleation density vs ion dosage. The ion energy is $150 \mathrm{eV}$.

induced nucleation enhancement for bombardment by $\left(\mathrm{CH}_{4}: \mathrm{Ar}: \mathrm{H}_{2}\right)$ ions. Above the threshold dose, the nucleation density increased remarkably to a maximum value of 3 $\times 10^{9} \mathrm{~cm}^{-2}$. A similar dependence of nucleation density on ion dose was obtained for the other three ion energies, although the density obtained at $10^{19}$ ions $\mathrm{cm}^{-2}$ appeared to show a slight tendency to decrease with decreasing ion energy.

For $\mathrm{Ar}^{+}$bombardment, the nucleation density shows merely a slight tendency toward increase. The highest nucleation density of $\sim 10^{6} \mathrm{~cm}^{-2}$ was obtained at a dose of 1 $\times 10^{19} \mathrm{~cm}^{-2}$, which is higher than the $\sim 10^{4} \mathrm{~cm}^{-2}$ normally obtained on a mirror-polished silicon substrate without any pretreatment. The increase of nucleation density may be caused by ion-bombardment-induced surface damages, which serve as the active sites for subsequent growth. This result is different from that of Kobayashi et al. ${ }^{26}$ who reported that no diamond could grow on a silicon substrate preimplanted with $100-\mathrm{keV}$ Ar ions at doses greater than 3 $\times 10^{15} \mathrm{~cm}^{-2}$. One logical explanation for the discrepancy is the difference in the ion energy used. For 100-keV Ar-ion implantation, most of the ion energy is deposited in the bulk. While in the present case of $100-\mathrm{eV}$ ions, energy is primarily deposited near the surface region, ${ }^{27}$ and the surface damage induced is responsible for the enhanced nucleation. Nevertheless, the enhancement induced by surface damages is limited, as the nucleation density is still two orders of magnitude lower than the value of $10^{8} \mathrm{~cm}^{-2}$ induced by conventional diamond powder scratching. When using $\mathrm{Ar}$ and $\mathrm{H}_{2}$ as the reactant gases $\left(\mathrm{Ar}: \mathrm{H}_{2}=1: 1\right)$, the nucleation density did not increase with ion dosage, but instead slightly decreased at high ion doses. The decrease of nucleation density may be due to the presence of hydrogen ions on the substrate surface, which removed the damages caused by $\mathrm{Ar}^{+}$bombardment. This result is similar to what was reported by Backmann et al. ${ }^{28}$ Addition of $\mathrm{CH}_{4}$ to the reactant gases gave strikingly different results. The nucleation density increases slightly with ion dosage from $10^{17}$ to $10^{18} \mathrm{~cm}^{-2}$; however, it increases sharply as the dosage increases from $10^{18}$ to $10^{19} \mathrm{~cm}^{-2}$. At $10^{19} \mathrm{~cm}^{-2}$ a nucleation density of about $10^{9} \mathrm{~cm}^{-2}$ was achieved. Compared to the case of only $\mathrm{Ar}$ gas, the addition of $\mathrm{CH}_{4}$ caused the nucleation density to increase by three orders of magnitude. This demonstrates that the nucleation enhancement by $\mathrm{Ar}^{+}$alone is negligible compared to that by the $\mathrm{CH}_{4} / \mathrm{H}_{2} / \mathrm{Ar}$ ions. Therefore, it is believed that hydrocarbon and hydrogen ions are primarily responsible for the nucleation enhancement in the ion bombardment process.

\section{B. SEM and Raman characterization of ion-deposited carbon films}

To investigate the mechanism of nucleation enhancement by hydrocarbon and hydrogen ion bombardment, the morphologies and phase compositions of the samples after bombardment at different ion doses and ion energies were studied by SEM, TEM and micro-Raman spectroscopy. Figures 5(a)-5(e) show SEM surface morphologies of the samples by $\left(\mathrm{CH}_{4}+\mathrm{H}_{2}+\mathrm{Ar}\right)$ ions at ion doses of $10^{17}, 5 \times 10^{17}, 10^{18}$, $5 \times 10^{18}$, and $10^{19} \mathrm{~cm}^{-2}$. The ion energy was $150 \mathrm{eV}$. For ion energies of 80,100 , and $200 \mathrm{eV}$, similar observations were obtained. When the ion dose was $10^{17} \mathrm{~cm}^{-2}$ [Fig. 5(a)], a smooth film was deposited, although some pinholes and dark regions due to ion etching could be observed. As the ion dose increased to $5 \times 10^{17} \mathrm{~cm}^{-2}$ [Fig. 5(b)], the surface of the sample was still smooth under SEM observation, but the area of dark regions increased. The appearance of dark regions was proposed to be due to the amassment of the ionbombardment-induced stress. As a result, the dark region increased with increasing ion dose. Increasing the ion dose further to $10^{18} \mathrm{~cm}^{-2}$ [Fig. 5(c)], the sample surface became rough. The dark regions still existed, but the average size of each region became smaller. Concurrently, many small clusters of 100-200 $\mathrm{nm}$ were formed on the surface. It was speculated that, when the stress in the dark regions accumulated to a sufficiently high level, the carbon species in the dark regions would aggregate to form clusters. As the ion dose reached $5 \times 10^{18} \mathrm{~cm}^{-2}$ [Fig. $\left.5(\mathrm{~d})\right]$, the dark regions became smaller but individual large clusters were formed. Finally, when the ion dose reached $10^{19} \mathrm{~cm}^{-2}$ [Fig. 5(e)], these clusters grew larger to 300-500 nm and the roughness of film surface significantly increased. It is interesting to compare the SEM observations with the results in Fig. 4. Just as the clusters started to form at the ion dose of $10^{18} \mathrm{~cm}^{-2}$ and enlarge with increasing ion dose, concurrently the diamond nucleation density in Fig. 4 also began to increase from an ion dose of $10^{18} \mathrm{~cm}^{-2}$.

Figure 6 shows the corresponding Raman spectra of the samples shown in Fig. 5. In the figure, two main peaks located at 1358 and $1588 \mathrm{~cm}^{-1}$, so-called $D$ and $G$ bands, ${ }^{29,30}$ can be observed in the spectra of all samples. The presence of the two bands shows that the deposited films were composed of amorphous carbon and graphite. The full width at half maximum (FWHM) of both the $D$ and $G$ bands decreased with increasing ion doses from $10^{17}$ to $10^{19} \mathrm{~cm}^{-2}$. For example, the FWHM is of the $D$ and $G$ bands were 272.7 and $127.3 \mathrm{~cm}^{-1}$, respectively at an ion dose of $10^{17} \mathrm{~cm}^{-2}$, 

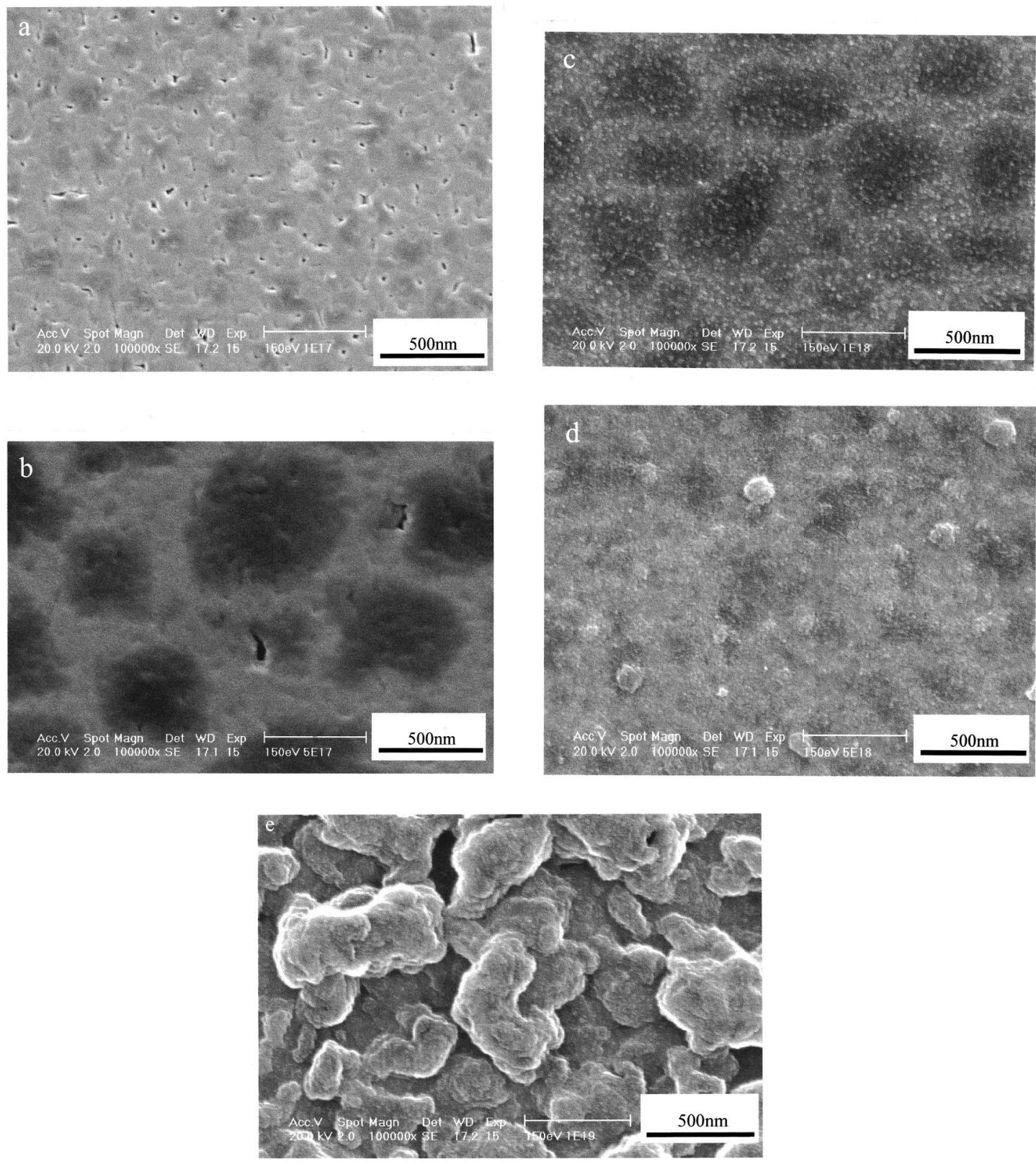

FIG. 5. SEM surface morphologies of the samples after ion bombardment by $150-\mathrm{eV}\left(\mathrm{CH}_{x}+\mathrm{H}_{x}+\mathrm{Ar}\right.$ ) ions with different doses: (a) $10^{17} \mathrm{~cm}^{-2}$, (b) $5 \times 10^{17} \mathrm{~cm}^{-2}$, (c) $10^{18} \mathrm{~cm}^{-2}$, (d) $5 \times 10^{18} \mathrm{~cm}^{-2}$, and (e) $10^{19} \mathrm{~cm}^{-2}$.

but decreased to 90.9 and $95.5 \mathrm{~cm}^{-1}$ at $10^{19} \mathrm{~cm}^{-2}$. The decrease of the FWHM's of both $D$ and $G$ bands is probably associated with the condensation or crystallization process of both amorphous carbon and graphite state with increasing ion dose. Furthermore, the ratio of the intensities of the $D$ band to the $G$ Band, usually referred as the $I(D) / I(G)$ ratio, ${ }^{31,32}$ increased with increasing ion dose. The $I(D) / I(G)$ ratio is known to increase with the size of the clusters in the film. ${ }^{30,33}$ Thus Raman observation shows that the crystal size increased with increasing ion dose. This is in accord with the SEM observation of surface morphologies in Fig. 5, where large clusters were observed at a high ion dose.

\section{Characterization of ion-deposited carbon film and $\mathrm{SiC}$ interlayer}

Figure 7(a) shows the TEM cross-sectional bright-field image of the sample bombarded at an ion dose of $10^{19} \mathrm{~cm}^{-2}$.
The ion energy was $100 \mathrm{eV}$. It can be seen that an amorphous carbon layer (marked as $C$ ) of thickness of $\sim 400 \mathrm{~nm}$ was deposited on the silicon substrate (marked as $A$ ). A SiC interlayer (marked as $B$ ) could be detected between the amorphous carbon film and silicon substrate. Some voids were observed on the substrate surface, most probably due to ion-induced etching. The corresponding SAED pattern of the sample in Fig. 7(a) is shown in Fig. 7(b). The diffraction pattern of $\mathrm{SiC}$ was observed to show an epitaxial relation with the silicon diffraction points. It is important to note that, in addition to the diffraction patterns of $\mathrm{Si}$ and $\mathrm{SiC}$ (indicated by a single narrow arrowhead), the $\{111\}$ and $\{220\}$ diffraction rings of diamond can also be observed, as indicated by double and single fat arrowheads, respectively. The formation of diamond is further confirmed by the HRTEM image taken from the amorphous film, which will be discussed later in the paper. 


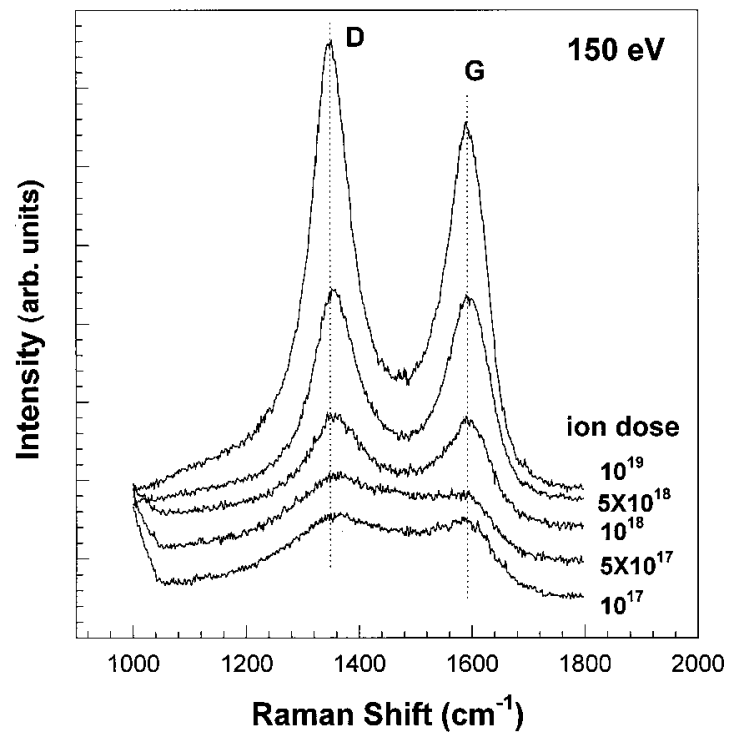

FIG. 6. Corresponding Raman spectra of samples in Figs. 5(a)$5(\mathrm{e})$.

The presence of $\mathrm{SiC}$ at the interface between the amorphous carbon layer and silicon substrate is demonstrated by the cross-sectional HRTEM shown in Fig. 8. The sample was bombarded with an ion dose of $10^{19} \mathrm{~cm}^{-2}$ at $80 \mathrm{eV}$. It revealed that an epitaxial $\mathrm{SiC}$ layer with a thickness of about $10 \mathrm{~nm}$ was formed between the amorphous carbon and silicon substrates. The epitaxial relation between the $\mathrm{SiC}$ and silicon substrate was also revealed by the SAED pattern in Fig. 7(b) for the sample after bombardment by ions of 100
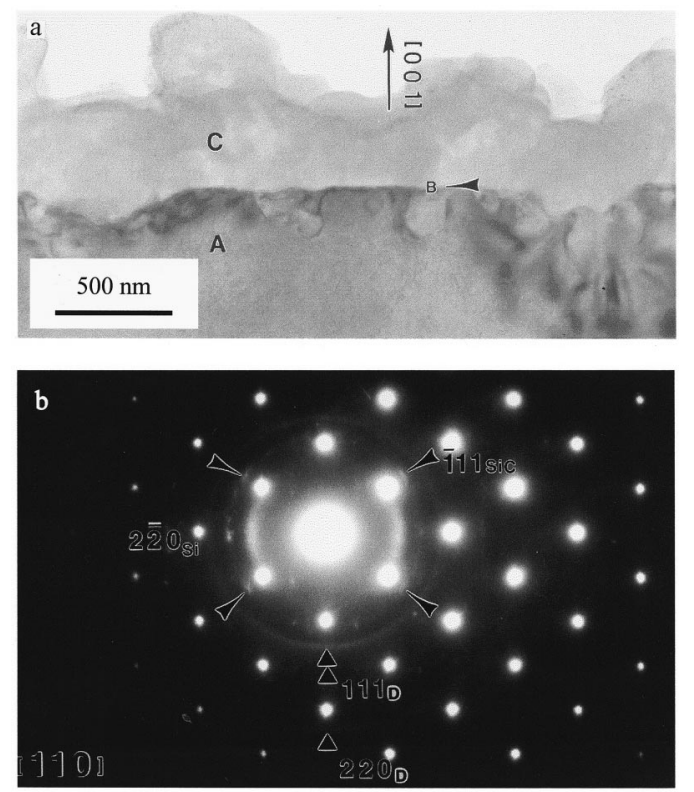

FIG. 7. (a) TEM cross-sectional bright-field image of the sample after ion bombardment at an ion dose of $10^{19} \mathrm{~cm}^{-2}$. The ion energy was $100 \mathrm{eV}$. The amorphous carbon film, $\mathrm{SiC}$ interlayer, and $\mathrm{Si}$ substrate are marked by $A, B$, and $C$, respectively. (b) The corresponding SAED pattern of the same sample in (a). The SiC $\{111\}$ reflections are marked by single thin arrows, while the diamond $\{111\}$ and $\{220\}$ reflections are marked by double and single fat arrows, respectively. The strong spots are the Si diffraction pattern.

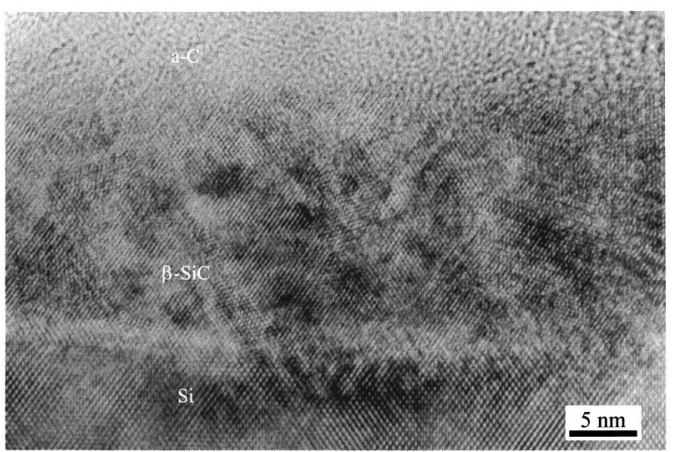

FIG. 8. HRTEM image taken near the interface between Si substrate and deposited amorphous carbon layer in the sample bombarded with an ion dose of $10^{19} \mathrm{~cm}^{-2}$. The ion energy is $80 \mathrm{eV}$.

$\mathrm{eV}$. Based on the experimental observations above, the following process can be reckoned to have happened during ion bombardment. The energetic hydrocarbon ions arriving at the substrate surface creating initially a $\mathrm{SiC}$ layer. As the bombardment continued, the energetic hydrocarbon clusters migrated and aggregated on the substrate surface, and subsequently an amorphous carbon layer was deposited on the $\mathrm{SiC}$ layer. This process was clearly revealed by Raman measurements on the sample after $200-\mathrm{eV}$ ion bombardment at a dose ranging from $10^{16}$ to $10^{19} \mathrm{~cm}^{-2}$, as shown in Fig. 9. At an ion dose of $10^{16} \mathrm{~cm}^{-2}$, only two peaks at about 820 and $980 \mathrm{~cm}^{-1}$ for $\mathrm{SiC}$ and $\mathrm{Si}$ can be observed. As the ion dose increased to $10^{17} \mathrm{~cm}^{-2}$, a broad signal centered at about $1470 \mathrm{~cm}^{-1}$ appeared, showing the presence of amorphous carbon on SiC. With increasing ion dose, the thickness of the amorphous film increased, so that the $\mathrm{SiC}$ signal gradually decreased and eventually disappeared at the ion dose of $10^{19} \mathrm{~cm}^{-2}$. Meanwhile, the $D$ and $G$ peaks increased at the expense of the broad signal at $1470 \mathrm{~cm}^{-1}$, and their FWHM decreased with increasing ion dose similar to what was described in Fig. 6. In addition to 80 and $100 \mathrm{eV}$, the $\mathrm{SiC}$ interface was also found in the samples after ion bombardment at 150 and $200 \mathrm{eV}$. However, the thickness and the

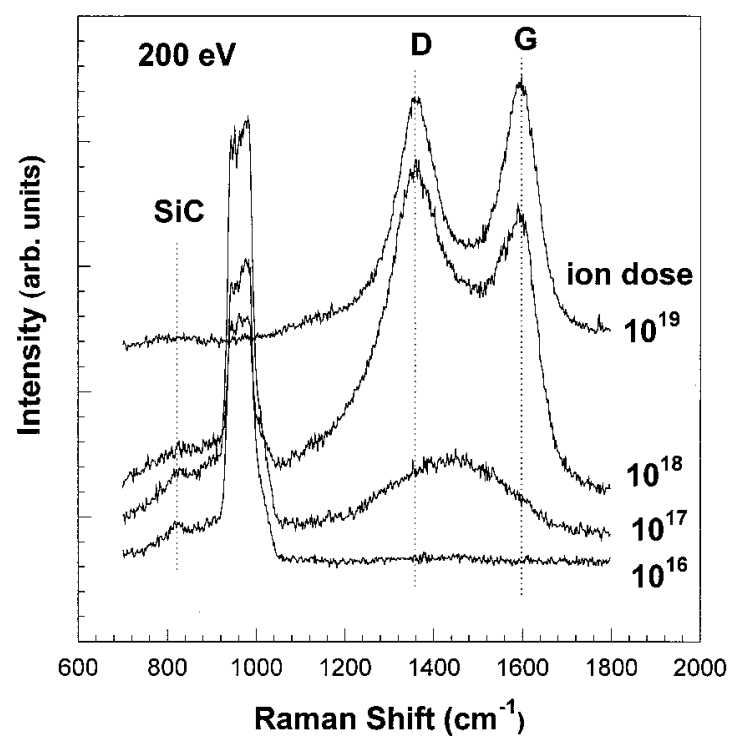

FIG. 9. Raman spectra of the sample after $200-\mathrm{eV}$ ion bombardment at an ion dose from $10^{16}$ to $10^{19} \mathrm{~cm}^{-2}$. 

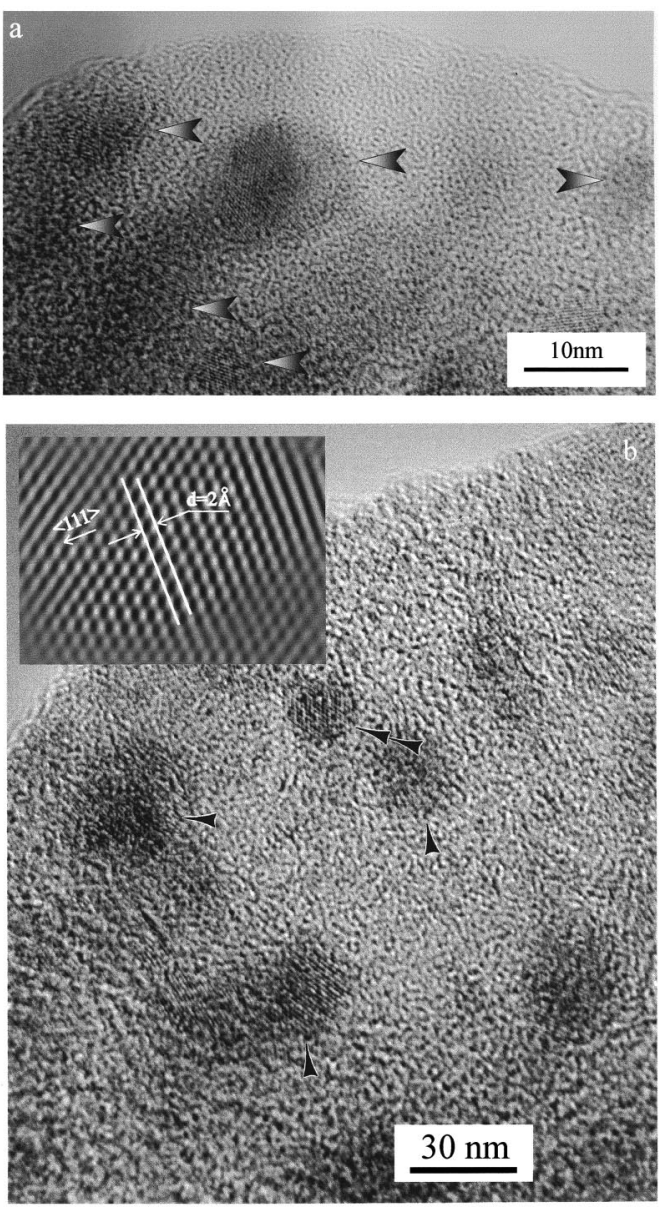

FIG. 10. HRTEM images of the amorphous carbon layer deposited by ion bombardment at an ion dose of (a) $1 \times 10^{18} \mathrm{~cm}^{-2}$ and (b) $10^{19} \mathrm{~cm}^{-2}$. Nanocrystalline diamond particles can be observed embedded in the matrix of amorphous carbon. The inset in (b) is the simulated image of the nanocrystals after image filter processing by Fourier transform.

epitaxial degree of the $\mathrm{SiC}$ layer decreased with increasing ion energy. Our results agree well with those of Ref. 11, where the subplantation efficiency was reported to reach a maximum for ion energies between 80 and $100 \mathrm{eV}$.

\section{Direct observation of diamond formation}

In addition to the diffraction pattern of diamond in the SAED pattern [Fig. 7(b)], the formation of diamond by the ion bombardment process was further confirmed by HRTEM observation, as shown in Fig. 10. The ion energy was chosen to be $150 \mathrm{eV}$, and the total ion dose varied from $10^{17}$ to $10^{19} \mathrm{~cm}^{-2}$. For ion doses below $1 \times 10^{18} \mathrm{~cm}^{-2}$, only an amorphous carbon phase was observed. Remarkably, when the ion dose reached $1 \times 10^{18} \mathrm{~cm}^{-2}$, a few randomly oriented nanocrystalline particles were found to be dispersed in the amorphous matrix, as shown in Fig. 10(a). These nanoparticles were 5-10 nm in size. At $5 \times 10^{18} \mathrm{~cm}^{-2}$, nanoparticles of 7-15 $\mathrm{nm}$ were found. As the ion dose increased to $10^{19} \mathrm{~cm}^{-2}$, the size of these nanoparticles increased to $20-30$ $\mathrm{nm}$, as shown in Fig. 10(b). After image filter processing by Fourier transform [see the inset in Fig. 10(b)], the lattice spacing of the nanocrystals was measured to be $2 \AA$, which matches well with the $d$-spacing of diamond $\{111\}$. Combining with the electron-diffraction pattern mentioned above, these nanoparticles are confirmed to be nanocrystalline diamond particles, and the $\langle 111\rangle$ directions of these particles are randomly oriented. It should be noted that only those particles with their $\{111\}$ crystal faces parallel to the electron beam direction can be observed in the HRTEM image. Thus the total density of the nanocrystalline particles in the amorphous matrix could possibly be higher than that observed in the HRTEM image. Compared with the SEM observation in Fig. 5 and the nucleation density dependence on ion dose (Fig. 4), it may be concluded that nucleation density was significantly increased only after nanocrystal diamond particles were formed.

\section{E. Ion-bombardment-induced diamond formation}

Thermal spikes ${ }^{34,35}$ and carbon subplantation ${ }^{36-40}$ are the two most popular models for nucleation enhancement by ion bombardment. According to the thermal spike model, it is difficult to explain why nanocrystalline diamond particles were observed only after the ion dose reached $10^{18} \mathrm{~cm}^{-2}$ in our experiments. Since the thermal spikes occurred from the beginning of the ion bombardment process, as a result nanocrystalline diamond would have appeared much earlier at smaller ion doses. The subplantation model, first suggested by Lifshitz and co-workers ${ }^{36,37}$ and used to explain the BEN process, ${ }^{41,42}$ is found more suitable to describe our present experiments. During ion bombardment, the energetic hydrocarbon ion arrives and enters the substrate surface; and eventually loses its energy in overcoming the penetration threshold potential. For low-energy ions $(\sim 100 \mathrm{eV})$ this is a major energy loss. Therefore, the ions are implanted within the top several layers of the substrate surface, and dissipate little energy before coming to rest in the film. A carbide layer is firstly formed on the substrate surface; afterwards a carbonrich film is deposited (either graphite or amorphous carbon). Because the implanted ions directly penetrate to an interstitial site or occupy a substitutional site, the local density of the bombarded region of the target increases, thus producing a compressive stress in the growing film. With more implanted ions, the density of the carbon film increases, and the ion-bombardment-induced internal stress gradually amasses and increases in the amorphous film; finally carbon clusters are produced. The compressive stress in the diamondlike carbon films deposited by ions was suggested to be possibly high enough for moving the phase stability line across from graphite to diamond in the carbon phase diagram. ${ }^{43}$ Interestingly, a graphite interface layer oriented with the $c$ axis in the surface, recently reported to be formed during the BEN process, was also though to be due to the compressive stress. ${ }^{44}$

When only methane and argon were used as the working gases, no diamond particles were observed in the deposited films. ${ }^{45}$ This is possibly due to the lack of sufficient hydrogen ions or atoms. Although there was a small amount of hydrogen ions or atoms from methane, the amount present might not be enough to stabilize the $s p^{3}$ component. When hydrogen was also used as a reactant gas, the amount of hydrogen atoms and ions was greatly increased. As a result, the $s p^{3}$-bonded clusters could grow larger and reach a critical size for forming stable diamond particles. Moreover, the 
high substrate temperature should also be an important factor in the formation of diamond particles. Results from modeling and computing the free energies of the crystal phase showed the critical size of diamond to decrease with increasing substrate temperature. ${ }^{46}$ The critical size was calculated to be $10.2 \mathrm{~nm}$ at room temperature and $4.8 \mathrm{~nm}$ at $800{ }^{\circ} \mathrm{C}$. Thus a high substrate temperature has been shown to favor diamond formation. Many researchers have reported the deposition of carbon films by ion beams; however, only amorphous films were observed. High substrate temperatures may have been an important factor for the formation of nanocrystalline diamond particles in our amorphous films.

The present work has clearly demonstrated that diamond particles can be produced by hydrogen/hydrocarbon ion bombardment. However, unlike the oriented diamond nuclei formed in the BEN process, only randomly oriented diamond particles were synthesized by direct ion bombardment. Furthermore, diamond particles were codeposited with a large amount of amorphous carbon. Two reasons may be contributing to the difference. First, the ion energy used in the present experiments was about $100 \mathrm{eV}$, which is much higher than the average ion energy of $\sim 20 \mathrm{eV}$ in the BEN process, as estimated in Ref. 47. Higher ion energy induces more damages to the substrate, which would hinder oriented diamond formation. Second, considering the ionization potential and ionization cross sections of methane, hydrogen, and argon, we estimated the doses of hydrocarbon and hydrogen ion in the present experiment to be $\sim 5 \times 10^{17}$ and $\sim 2 \times 10^{18} \mathrm{~cm}^{-2}$, respectively. Compared with the BEN process, where the total dose of hydrocarbon and hydrogen ions was approximately $1 \times 10^{20} \mathrm{~cm}^{-2}$ under the conventional experimental conditions, i.e., substrate bias was applied on a 2 -in. Si wafer at a current of about $40 \mathrm{~mA}$ for $15 \mathrm{~min}$. Thus the ion dose used in the present study is two orders of magnitude lower. As a result, we suspect that decreasing the ion energy and increasing the ion dosage in the ion bombardment process could increase the orientation degree of diamond particles and the film purity. Unfortunately, the minimum ion energy is limited to $60 \mathrm{eV}$ in the present ion beam system, in which it already took nearly $10 \mathrm{~h}$ to obtain a dosage of $1 \times 10^{19} \mathrm{~cm}^{-2}$.

When CVD was subsequently performed on the ionbombarded sample, the amorphous carbon was preferentially etched away by atomic hydrogen in the CVD plasma, and the nanocrystalline diamond particles exposed. The exposed particles could possibly serve as nuclei in the subsequent diamond growth. Remarkably, the nucleation density counted after 15-min growth (Fig. 3) is one order of magnitude lower than the nanoparticle density of about $10^{10} \mathrm{~cm}^{-2}$ in the amorphous carbon film [Fig. 10(b)]. The observation suggests that most of the nanocrystalline diamond particles did not survive in the growth environment, and only a very small portion $(<10 \%)$ of the particles could grow and serve as nuclei in the growth process. The cross-sectional TEM image of the sample bombarded with an ion dose of $10^{19} \mathrm{~cm}^{-2}$ (the ion energy was $150 \mathrm{eV}$ ) and grown by HFCVD for $15 \mathrm{~min}$ is shown in Fig. 11. Some diamond grains of more than $100 \mathrm{~nm}$ in size are now directly located on the silicon substrate surface, whereas the amorphous carbon surrounding the nanocrystal diamond particles have been etched away. This ob-

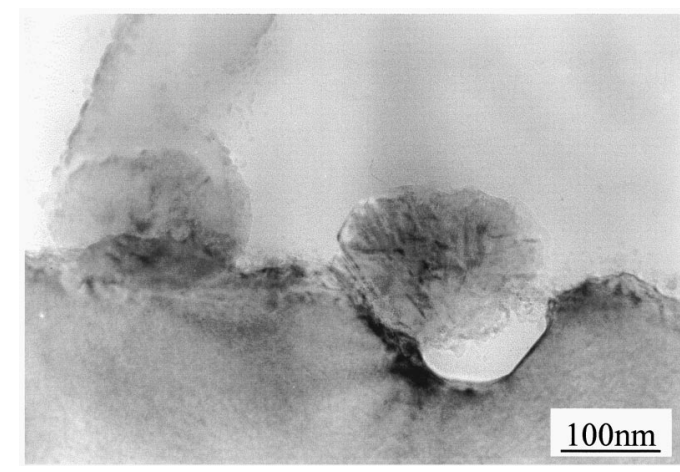

FIG. 11. TEM cross-sectional bright-field image of the sample after ion bombardment at an ion dose of $10^{19} \mathrm{~cm}^{-2}$, and subsequent deposition by conventional HFCVD for $15 \mathrm{~min}$. The ion energy was $150 \mathrm{eV}$.

servation is consistent with the removal of amorphous carbon and the growth of diamond nanoparticles during the HFCVD process.

\section{CONCLUSIONS}

The effects of ion bombardment on diamond nucleation were directly studied by the ion beam deposition technique with $\mathrm{Ar} / \mathrm{H}_{2} / \mathrm{CH}_{4}$ as the reactant gases. We demonstrated that direct ion beam bombardment can be successfully applied for the nucleation of nanodiamond crystallites on $\mathrm{Si}(100)$ substrates. These nanodiamond particles were further proposed to serve as nuclei for the subsequent growth process by conventional HFCVD. The nucleation density increased with increasing ion dose bombarding the substrate. A nucleation density higher than $10^{9} \mathrm{~cm}^{-2}$ was achieved with an ion dose of $10^{19} \mathrm{~cm}^{-2}$. The observations were found very helpful for the evaluation of the mechanism of bias-enhanced nucleation of diamond.

Detailed investigation of the deposited film showed that the ion bombardment process induced the formation of a $\mathrm{SiC}$ layer on the silicon substrate. The quality and thickness of the $\mathrm{SiC}$ layer depended strongly on the ion energy. At an ion energy of $80 \mathrm{eV}$, an epitaxial $\mathrm{SiC}$ layer of $10 \mathrm{~nm}$ was formed. With increasing ion energy, the thickness of the $\mathrm{SiC}$ as well as its degree of epitaxial alignment with the Si substrate decreased. Continuing the ion bombardment, an amorphous carbon film was eventually deposited on the $\mathrm{SiC}$ layer. The amorphous carbon film condensed to form clusters, and the film surface became rougher with increasing ion dose. At high doses $\left(>10^{18} \mathrm{~cm}^{-2}\right)$, nanocrystalline diamond particles were detected in the amorphous carbon film, and the size of the diamond particles increased with increasing ion dose. The formation of nanocrystalline diamond particles is proposed to be due to the ion-bombardment-induced stress. The presence of hydrogen ions in the ion bombardment process also played a critical role in the formation of nanocrystalline diamond particles.

\section{ACKNOWLEDGMENTS}

The Research Grant Council of Hong Kong and Strategic Research Grants of the City University of Hong Kong supported this project. 
* Author to whom all correspondence should be addressed. Electronic address: apannale@ cityu.edu.hk

${ }^{1}$ K. Ishibashi and S. Furukawa, Jpn. J. Appl. Phys., Part 1 24, 912 (1985).

${ }^{2}$ W. A. Yarbrough and R. Messier, Science 247, 688 (1990).

${ }^{3}$ Jie Yang, Xiaowei Su, Qijin Chen, and Zhangda Lin, Appl. Phys. Lett. 66, 3284 (1995).

${ }^{4}$ S. Yugo, T. Kanai, T. Kimura, and T. Muto, Appl. Phys. Lett. 58, 1036 (1991).

${ }^{5}$ B. R. Stoner and J. T. Glass, Appl. Phys. Lett. 60, 698 (1992).

${ }^{6}$ X. Jiang and C.-P. Klages, Diamond Relat. Mater. 1, 195 (1992).

${ }^{7}$ J. Yang, Z. Lin, L.-X. Wang, S. Jin, and Z. Zhang, Appl. Phys. Lett. 65, 3203 (1994).

${ }^{8}$ F. Stubhan, M. Ferguson, H. J. Füsser, and R. J. Behm, Appl. Phys. Lett. 66, 1900 (1995).

${ }^{9}$ S. Yugo, T. Kimura, and T. Kanai, Diamond Relat. Mater. 2, 328 (1993).

${ }^{10}$ S. Yugo, N. Nakamura, and T. Kimura, Diamond Relat. Mater. 7, 1017 (1998).

${ }^{11}$ J. Gerber, S. Sattel, K. Jung, H. Ehrhardt, and J. Robertson, Diamond Relat. Mater. 4, 559 (1995).

${ }^{12}$ B. R. Stoner, G. J. Tessmer, and D. L. Dreifus, Appl. Phys. Lett. 62, 1803 (1993).

${ }^{13}$ J. S. Lee, K. S. Liu, and I.-N. Lin, Appl. Phys. Lett. 67, 1555 (1995).

${ }^{14}$ Y. Shigesato, R. E. Boekenhauer, and B. W. Sheldon, Appl. Phys. Lett. 63, 314 (1993).

${ }^{15}$ R. Beckmann, B. Sobisch, W. Kulisch, and C. Rau, Diamond Relat. Mater. 3, 555 (1994).

${ }^{16}$ X. Jiang, K. Schiffmann, and C.-P. Klages, Phys. Rev. B 50, 8402 (1994).

${ }^{17}$ M. Tomellini, P. Polini, and V. Sessa, J. Appl. Phys. 70, 7573 (1991)

${ }^{18}$ X. Jiang, W. J. Zhang, M. Paul, and C.-P. Klages, Appl. Phys. Lett. 68, 1927 (1996).

${ }^{19}$ X. Jiang, W. J. Zhang, and C.-P. Klages, Phys. Rev. B 58, 7064 (1998).

${ }^{20}$ S.-T. Lee, W. M. Lau, L. J. Huang, Z. Ren, and F. Qin, Diamond Relat. Mater. 7, 96 (1995).

${ }^{21}$ I. M. Buckly-Golder, R. Bullough, M. R. Hayns, J. R. Willis, R. C. Piller, N. G. Blamires, G. Gard, and J. Stephen, Diamond Relat. Mater. 1, 43 (1991)

${ }^{22}$ M. Kitabatake and K. Wasa, J. Appl. Phys. 58, 1693 (1985).

${ }^{23}$ J. F. Prins and H. L. Maipher, in New Diamond Science and
Technology, edited by R. Messier and J. T. Glass (Materials Research Society, Pittsburgh, 1991), p. 561.

${ }^{24}$ S. T. Lee, S. Chen, G. Braunstein, X. Feng, I. Bello, and W. M. Lau, Appl. Phys. Lett. 59, 785 (1991).

${ }^{25}$ Y. P. Guo, K. L. Lam, K. M. Lui, R. W. M. Kwok, and K. C. Hui, J. Mater. Res. 13, 2315 (1998).

${ }^{26}$ K. Kobayashi, M. Kumagai, S. Karasawa, T. Watanabe, and F. Togashi, J. Cryst. Growth 128, 408 (1993).

${ }^{27}$ S. Mohajerzadeh and C. R. Selvakumar, Appl. Phys. Lett. 81, 3003 (1997).

${ }^{28}$ P. K. Backmann, R. Weimer, W. Drawl, Y. Liou, and R. Messier (unpublished).

${ }^{29}$ M. Yoshikawa, Mater. Sci. Forum 52-53, 365 (1989).

${ }^{30}$ M. A. Tamor and V. C. Vassell, J. Appl. Phys. 76, 3823 (1994).

${ }^{31}$ F. Tunistra and J. K. Koenig, J. Chem. Phys. 53, 1126 (1970).

${ }^{32}$ L. Yu, Khriachtchev, M. Hakovirta, R. Lappalainen, and M. Räsänen, Phys. Lett. A 217, 354 (1996).

${ }^{33}$ J. Robertson, Surf. Coat. Technol. 50, 185 (1992).

${ }^{34}$ F. Seitz and J. Kohler, in Progress in Solid State Physics, edited by F. Seitz and D. Turnbull (Academic, New York, 1956).

${ }^{35}$ C. Weissmantel, in Thin Films from Free Atoms and Particles, edited by K. Klabunde (Academic, Orlando, FL, 1985).

${ }^{36}$ Y. Lifshitz, S. R. Kasi, and J. W. Rabalais, Phys. Rev. Lett. 62, 1290 (1989).

${ }^{37}$ Y. Lifshitz, G. Lempert, and E. Grossman, Phys. Rev. Lett. 72, 2753 (1994).

${ }^{38}$ J. Robertson, Diamond Relat. Mater. 4, 549 (1995).

${ }^{39}$ J. Robertson, J. Gerber, S. Sattel, M. Weiler, K. Jung, and H. Ehrhardt, Appl. Phys. Lett. 66, 3287 (1995).

${ }^{40}$ J. Gerber, M. Weiler, O. Sorh, K. Jung, and H. Ehrhardt, Diamond Relat. Mater. 3, 506 (1994).

${ }^{41}$ S. P. McGinnis, M. A. Kelly, and S. B. Hagstrom, J. Appl. Phys. 79, 170 (1997).

${ }^{42}$ S. P. McGinnis, M. A. Kelly, and S. B. Hagstrom, J. Mater. Res. 12, 3354 (1997).

${ }^{43}$ Y. Lifshitz, S. Kasi, J. Rabalais, and W. Eckstein, Phys. Rev. B 41, 10468 (1990).

${ }^{44}$ I. Jiménez, M. Mar García, J. M. Albella, and L. J. Terminello, Appl. Phys. Lett. 73, 2911 (1998).

${ }^{45}$ S. T. Lee, S. Chen, J. Agostinelli, and G. Braustein, Appl. Phys. Lett. 60, 2213 (1992).

${ }^{46}$ M. Y. Gamarnik, Nanostruct. Mater. 7, 651 (1996).

${ }^{47}$ W. Kulisch, L. Ackermann, and B. Sobisch, Phys. Status Solidi A 154, 155 (1996). 